\title{
Magnetic Field Dependence of Transition to High Electron Density Phase in a Linear Plasma Device NUMBER ${ }^{*}$
}

\author{
Hiroki HACHIKUBO, Atsushi OKAMOTO, Takaaki FUJITA, Hideki ARIMOTO, Ryosuke OCHIAI, \\ Minami SUGIMOTO and Kento IIZUKA \\ Graduate School of Engineering, Nagoya University, Furo-cho, Chikusa-ku, Nagoya 464-8603, Japan
}

(Received 6 January 2020 / Accepted 10 May 2020)

\begin{abstract}
High electron density discharge mode was observed in the downstream of a magnetic beach, so called test region in a linear plasma device with an electron cyclotron resonance source. A model for formation of the high density phase based on electron motion along the field line was proposed. The model reproduced the tendency that the duration of the high density phase was longer for the higher magnetic field in the test region in the experiment. Some discrepancies between the experiment and the model were newly found in the experiment with detailed scan in the magnetic field in the test region with the modified time evolution of the magnetic field.
\end{abstract}

(C) 2020 The Japan Society of Plasma Science and Nuclear Fusion Research

Keywords: linear plasma device, electron cyclotron resonance, ion saturation current, magnetic mirror effect

DOI: $10.1585 /$ pfr. 15.2401042

\section{Introduction}

Cylindrical laboratory plasmas are widely utilized for studies on divertor plasma physics [1-4] and waveparticle interaction physics [5]. These studies are sometimes performed with high density plasmas of the order of $\sim 10^{18} \mathrm{~m}^{-3}$. In Nagoya University, a magnetoplasma basic experiment device, NUMBER, was developed for such purposes with the aid of electron cyclotron resonance by a microwave injected along the external magnetic field. Then an overdense plasma [6] is suitably produced. While the external magnetic field for plasma production is constrained under the resonance condition, arbitrary strength of magnetic field is applicable in the downstream of a magnetic beach, so called the test region. Then, a high density plasma in magnetic field up to $0.3 \mathrm{~T}$ is supplied for the study of basic plasma physics described above.

In previous research [7], an increase/decrease of the ion saturation current was observed with a Langmuir probe. The changes in the ion saturation current was caused by the changes in electron density while the electron temperature was unchanged. Formation of the high electron density phase depended on magnetic field in the test region and on the gas pressure. Recently, a simple model describing the high electron density phase was proposed [8], where a temporal evolution of the ion saturation current observed in the experiment was reproduced by the model. On the other hand, systematic comparison between experiments and the model in wide ranges of parameters is needed for better understanding of this phenomenon and for utilizing it for higher density plasma environment.

Toward this goal, we investigated the ion saturation

author'se-mail: okamoto.atsushi@nagoya-u.jp

*) This article is based on the presentation at the 28th International Toki Conference on Plasma and Fusion Research (ITC28). current dependence on the magnetic field in this study. Conditions for transition between the high and low density phases were compared between experiments and the model. For that reasons, new time evolution of magnetic field in the test region was developed to create a quasisteady state. Experimental setup is explained in Sec. 2, followed by a brief description of the simple model of the high density phase formation in Sec.3. Results are shown and discussed in Sec. 4. The paper is summarized in Sec. 5.

\section{Experimental Setup}

The experimental device NUMBER consists of the production region $(0<z<0.7 \mathrm{~m})$ and the test region $(1.1 \mathrm{~m}<z<1.8 \mathrm{~m})$ as shown in Fig. 1 (a), where $z$ is a distance from the microwave injection window. A vacuum chamber made of stainless steel has dimensions of $0.2 \mathrm{~m}$
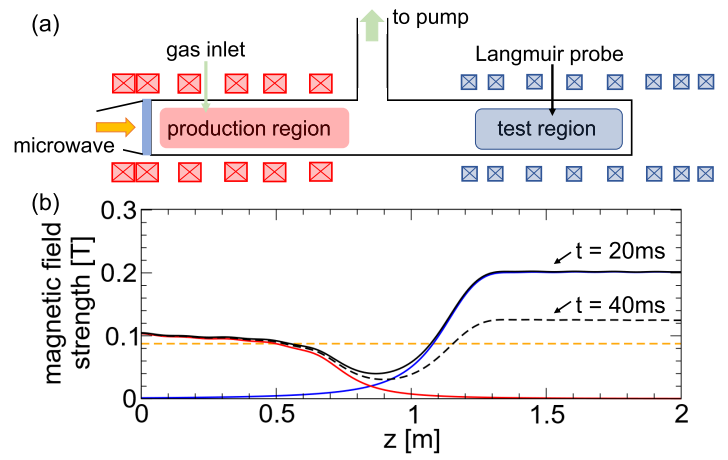

Fig. 1 Schematic of (a) experimental setup and (b) magnetic field strength on the axis in NUMBER at times $t=20 \mathrm{~ms}$ and $40 \mathrm{~ms}$ from the pulse magnetic field in the test region turns on. The horizontal orange broken line indicates the magnetic field, $B_{\mathrm{ECR}}=0.0875 \mathrm{~T}$. 
in diameter and $1.8 \mathrm{~m}$ in axial length. Helium gas is used in the experiments. Operation gas pressure is about $0.2 \mathrm{~Pa}$. In Fig. 1 (b), the red solid curve indicates the magnetic field generated by a DC power supply, the blue solid curve indicates the magnetic field generated by a capacitor bank at $t$ $=20 \mathrm{~ms}$ and the horizontal orange broken line indicates the magnetic field for the electron cyclotron resonance (ECR) with $2.45 \mathrm{GHz}$ microwave, $B_{\mathrm{ECR}}=0.0875 \mathrm{~T}$.

Power supply for generating magnetic field in the production region is in steady state operation. As shown in Fig. 1 (b), the magnetic field configuration is so called magnetic beach; the magnetic field strength is maximum at an end, where the microwave is injected, then it is gradually decreases along the chamber axis. The plasma is produced by ECR with a microwave, which is injected along the magnetic field through a quartz window from the end of the device. The frequency and injection power of a microwave power source are $2.45 \mathrm{GHz}$ and $6.0 \mathrm{~kW}$, respectively. Because $B_{\mathrm{ECR}}=0.0875 \mathrm{~T}$ is the resonance magnetic field strength, the magnetic field at the injection window, $B_{\text {prod. }}$, should satisfy a condition $B_{\text {prod. }}>B_{\mathrm{ECR}}$ for the overdense plasma production. The resonance magnetic field $B_{\mathrm{ECR}}$ arises at about $z=0.6 \mathrm{~m}$ from the injection window.

The magnetic field in the test region $B_{\text {test }}$ is uniform and is applied up to $0.3 \mathrm{~T}$. Then a local minimum of magnetic field exists between these two regions making a magnetic mirror.

The magnetic coil current for the test region is supplied by a two-stage capacitor bank network. In the present experiment, two discharge waveforms for the capacitor bank are used. One is a normal operation. Waveform is shown in Fig. 2. The magnetic field strength reaches maximum about $t=12 \mathrm{~ms}$ after the circuit is turned on. Then it gradually decreases. In Fig. 1 (b), the black solid and dotted curves indicate the total magnetic field at $t=20 \mathrm{~ms}$ and $40 \mathrm{~ms}$, respectively.

The other discharge waveform is labeled "new" in Fig. 2. The magnetic field strength has a quasi-steady state or a flat section in time evolution. By adjusting the magnetic field strength in flat section, $B_{\text {flat }}$, we can obtain slow variation of the magnetic field in the test region. We defined magnetic field in the test region at $t=40 \mathrm{~ms}$ as $B_{\text {flat }}$. We got a flat duration of $7 \mathrm{~ms}$ which was defined as the

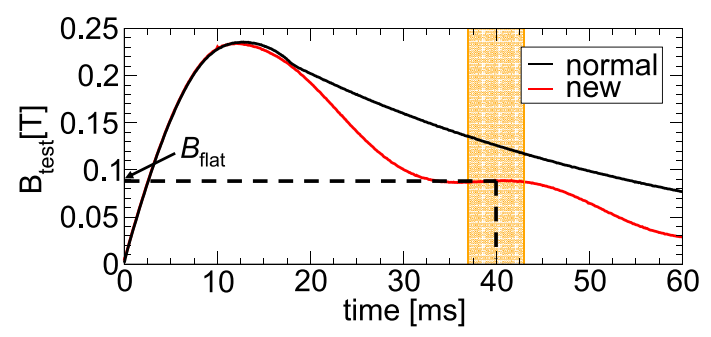

Fig. 2 Time evolution of the magnetic field in the test region for normal operation (black) and new operation (red). An orange hatch indicates a flat duration of $7 \mathrm{~ms}$. period that magnetic field changed within $\pm 1 \%$ around the value at $t=40 \mathrm{~ms}$.

A Langmuir probe is inserted in the test region $(z=$ $1.53 \mathrm{~m}$ ) to measure the ion saturation current. Typical electron density and temperature are $n_{\mathrm{e}}=5-10 \times 10^{17} \mathrm{~m}^{-3}$ and $T_{\mathrm{e}}=4 \mathrm{eV}$ in the test region for high density phase, while $n_{\mathrm{e}}<1 \times 10^{17} \mathrm{~m}^{-3}$ and $T_{\mathrm{e}}=4 \mathrm{eV}$ for low density phase.

\section{A Simple Model}

Here a simple model for formation of the high density phase [8] is briefly described. In this model that estimate electron influx from electrons motion in the direction of magnetic field, the electron influx in the test region is evaluated considering effects of the magnetic mirror between the production region and the test region. We categorized electron orbits in the magnetic field into case (i) to case (vi) as shown in Fig. 3. The horizontal red, orange and blue broken lines indicate the magnetic field $B_{\text {prod. }}, B_{\mathrm{ECR}}$ and $B_{\text {test }}$, respectively. The vertical black broken line indicates Langmuir probe position in the test region.

Definition of each case of electron orbit is shown in Table 1 . We classify whether electrons can pass through magnetic mirrors in the production region and in the test region. The second column of Table 1 shows whether or not electrons contribute to ionization. The third column shows whether or not magnetic mirror in the production region reflects the electron. The fourth column shows whether or not magnetic mirror in the test region reflects the electron.

The cases (i) - (vi) represent a characteristic pitch angle $\chi$ of electron at a minimum magnetic field $B_{\min }$, where $\cos \chi=v_{/ /} / v$ and $v_{/ /}$is the parallel velocity of the electron. Electrons in case (i) correspond to $\sin ^{2} \chi<$ $B_{\min } / \max \left(B_{\text {prod }}, B_{\text {test }}\right)$ and are neither reflected by magnetic mirror in the production region nor by magnetic mirror in the test region. So, electrons in the case (i) freely escape to the end of the production region and that of the test region. Electrons in case (ii) correspond to $B_{\min } / B_{\text {test }}<$
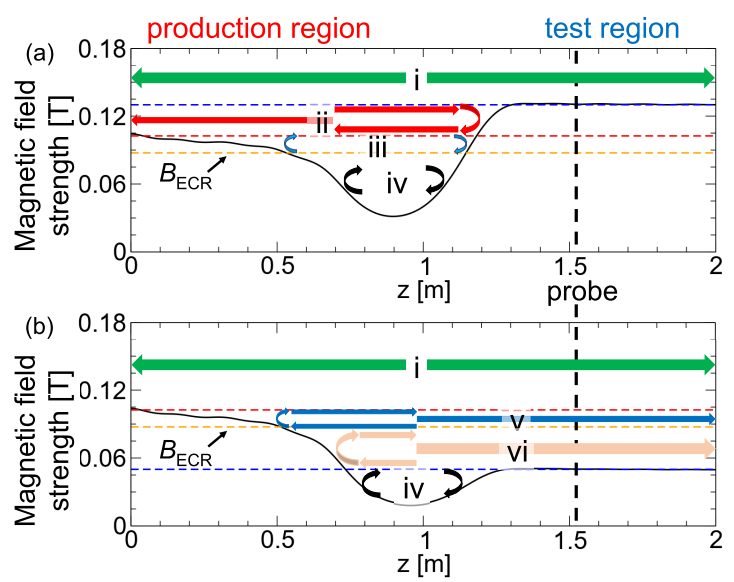

Fig. 3 Magnetic field strength and behavior of electron orbits in normal discharge operation at (a) $40 \mathrm{~ms}$ and (b) $75 \mathrm{~ms}$. 
Table 1 Classification of electron orbits from case (i) to case (vi).

\begin{tabular}{llll}
\hline case & $\begin{array}{l}\text { Contribute } \\
\text { to ionization }\end{array}$ & $\begin{array}{l}\text { Reflection } \\
\text { (prod. region) }\end{array}$ & $\begin{array}{l}\text { Reflection } \\
\text { (test region) }\end{array}$ \\
\hline (i) & No & No & No \\
(ii) & No & No & Yes \\
(iii) & Yes & Yes & Yes \\
(iv) & No & Yes & Yes \\
(v) & Yes & Yes & No \\
(vi) & No & Yes & No \\
\hline
\end{tabular}

$\sin ^{2} \chi<B_{\min } / B_{\text {prod }}$ and are not reflected by magnetic mirror in the production region but reflected by magnetic mirror in the test region. So, electrons in the case (ii) are prohibited to enter into the test region due to the magnetic mirror in front of the test region. Electrons in case (iii) $\left(B_{\min } / B_{\text {prod }}<\sin ^{2} \chi<B_{\min } / B_{\mathrm{ECR}}\right)$ and case (iv) $\left(\sin ^{2} \chi>\right.$ $\left.B_{\min } / \min \left(B_{\mathrm{ECR}}, B_{\text {test }}\right)\right)$ are trapped both by magnetic mirror in the production region and by magnetic mirror in the test region. So, the electrons of case (iv) are mirror trapped without resonance condition, which does not contribute to particle supplying to the test region nor to ionization. Electrons in case $(\mathrm{v})\left(B_{\min } / B_{\mathrm{ECR}}<\sin ^{2} \chi<B_{\min } / B_{\text {test }}\right)$ and case (vi) $\left(\sin ^{2} \chi>B_{\min } / B_{\mathrm{ECR}}\right)$ are reflected by magnetic mirror in the production region but not reflected by magnetic mirror in the test region. So, electrons in the case (vi) escape only to the test region, and no resonance condition.

Electrons in case (iv) and case (vi) do not satisfy the ECR condition. Electrons in other cases satisfy the ECR condition. In the latter electrons, those in case (iii) are confined by mirrors. Hence the electrons in case (iii) efficiently gain energy from the microwave and contribute to ionization. Electrons in case (i) and case (ii) are quickly lost at the microwave injection window and hence they are considered to contribute little to ionization. Electrons in case (v), which does not reach the microwave injection window and reach the wall at the end of the test region, are considered to gain energy from the microwave but smaller efficiency on ionization than electrons in case (iii). The electrons of cases (iii) and (v) contribute to plasma production or electron collision ionization. Figure 4 shows time evolution of fractions of electron orbits for the magnetic field at the test regions with $B_{\max }=0.23 \mathrm{~T}$ of the normal operation. We obtain a temporal variation of those fractions at the minimum magnetic field position under the assumption of the isotropic distribution.

Considering the properties of the electron orbits described above we calculated electron influx in the test region by $(f($ iii $)+0.1 f(\mathrm{v})) \times(0.5 f(\mathrm{i})+f(\mathrm{v})+f(\mathrm{vi}))$ in the model [8], where $f(\bullet)$ denotes the fractions of electrons included in each case. The former term indicates electrons that contribute to ionization. Electrons that contribute to ionization are case (iii) and case (v); $100 \%$ and $10 \%$ ion-

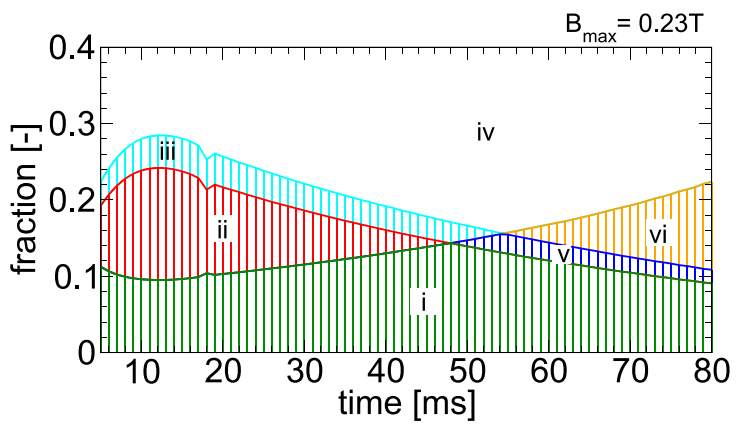

Fig. 4 Cumulative fraction of electron orbits based on the magnetic field variation in the experiment $\left(B_{\max }=0.23 \mathrm{~T}\right)$.

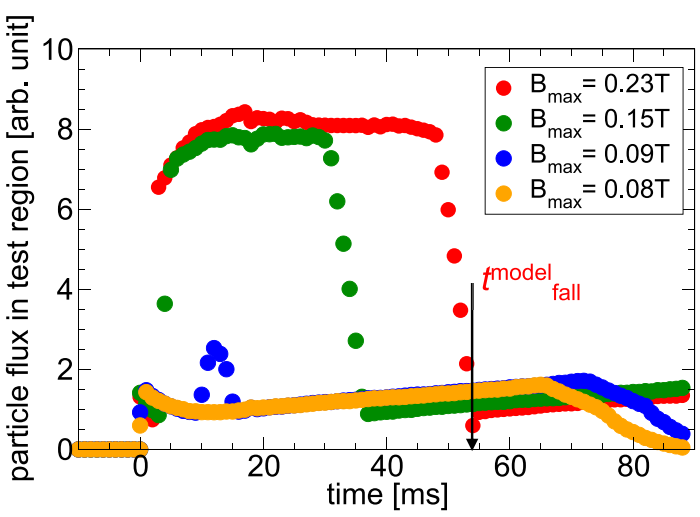

Fig. 5 Time evolution of electron influx in the test region.

ization probabilities are assumed. In the latter term, case (v), case (vi) and half of case (i) are transported into the test region [8]. Then, we can obtain the time evolution of electron influx as shown in Fig. 5.

\section{Results and Discussion}

\subsection{Dependence of the ion saturation cur- rent on magnetic field in the test region}

We investigated dependence of ion saturation current $I_{\text {is }}$ on the magnetic field $B_{\text {test }}$ for various maximum magnetic fields. Figure 6 shows time evolution of $B_{\text {test }}$ and $I_{\text {is }}$ in the test region. $B_{\text {test }}$ was scanned by changing the charging voltage of the capacitor bank. The maximum value of $B_{\text {test }}$ during the pulse is denoted by $B_{\max }$ in this article. Before the circuit for the magnetic field in the test region is turned on $(t=0), I_{\text {is }}$ is nearly zero, which indicates negligibly low density plasma exists in the test region. $I_{\text {is }}$ began to rise at $t=0$. For two cases with $B_{\max }=0.23 \mathrm{~T}$ and $0.15 \mathrm{~T}$, the rising rate of $I_{\text {is }}$ was largely increased around $t=7 \mathrm{~ms}$ and $I_{\text {is }}$ reached a high level and was kept nearly constant before dropping toward the lower level. The duration that $I_{\text {is }}$ was kept at a higher level is called the high electron density phase.

The duration of the high density phase is longer at $B_{\max }=0.23 \mathrm{~T}$ than at $B_{\max }=0.15 \mathrm{~T}$. A short high den- 

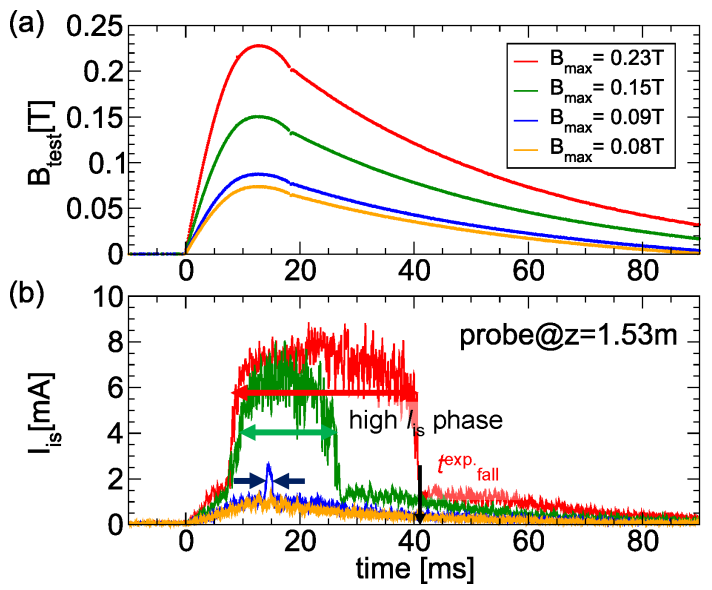

Fig. 6 Time evolution of (a) $B_{\text {test }}$ and (b) $I_{\text {is }}$.

sity phase was observed at $B_{\max }=0.09 \mathrm{~T}$, but not at $B_{\max }$ $=0.08 \mathrm{~T}$. So, it seems that a threshold exists in $B_{\text {test }}$ for formation of the high density phase.

\subsection{Model for the high density phase consid- ering magnetic mirror effect}

We compared electron influx in the test region in the model shown in Fig. 5 and measurement of $I_{\text {is }}$ in the experiment shown in Fig. 6(b). The latter is a good measure of the electron influx because of the quasi-neutrality. We defined the time at a rapid decrease of electron influx ( $\left.t_{\text {fall }}^{\text {molel }}\right)$ and $I_{\text {is }}\left(t_{\text {fall }}^{\text {exp. }}\right)$ in the test region as shown in Fig. 5 and Fig. 6 (b) for $B_{\max }=0.23 \mathrm{~T}$. It is found that the model agrees with the experiment on the high density phase formation and tendency of the duration. The electron influxes are almost the same during the high density phase both for $B_{\max }=0.15$ and $0.23 \mathrm{~T}$ cases in Fig. 5. This is similar to behavior of $I_{\text {is }}$ observed in Fig. 6 (b). For a marginal condition $B_{\max }=0.09 \mathrm{~T}$, a smaller peak with short duration is observed both in the model and the experiment. On the other hand, quantitative difference is shown between $t_{\text {fall }}^{\text {exp. }}$ and $t_{\text {fall }}^{\text {model }}$. We defined $B_{\text {test }}$ at a rapid decrease of electron influx in the test region as $B_{\text {fall }}^{\text {model }}$ and that at a rapid

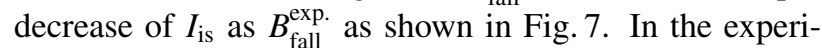

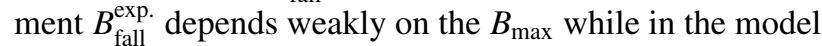
$B_{\text {fall }}^{\text {molel }}$ does not depend on the $B_{\text {max }}$. $B_{\text {fall }}$ is different in the experiment and in the model.

\subsection{Measurement of the ion saturation cur- rent in the modified magnetic field in the test region}

In order to observe small changes of $B_{\text {fall }}$, the new time evolution of $B_{\text {test }}$ to create a flat section was applied. We measured $I_{\text {is }}$ in the test region for various magnetic field strength in the test region.

Figure 8 shows the fall time of $I_{\text {is }}\left(t_{\text {fall }}^{\text {exp. }}\right)$ and that of the electron influx in the test region ( $\left.t_{\text {fall }}^{\text {model }}\right)$ as functions of $B_{\text {flat }}$, for magnetic field at the end of the production region $B_{\text {prod. }}=0.100 \mathrm{~T}$ and $0.103 \mathrm{~T}$. The fall time is later
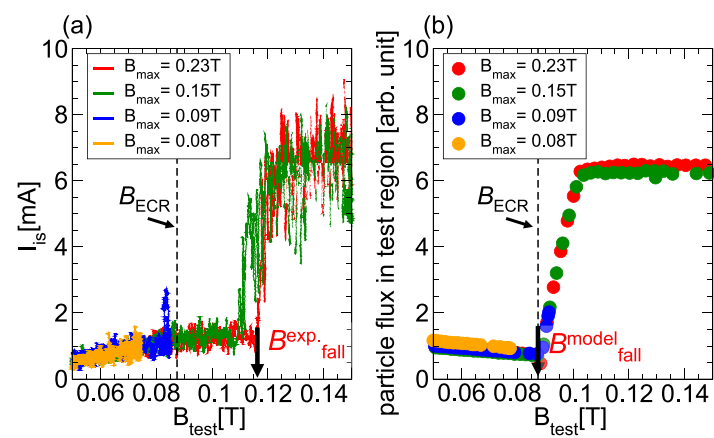

Fig. 7 A rapid decrease of (a) $I_{\text {is }}$ and (b) particle flux in the test region as a function of $B_{\text {test }}$.

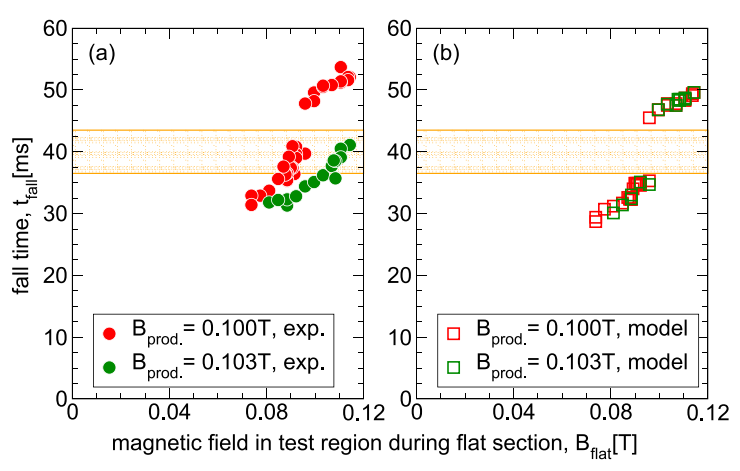

Fig. 8 The fall time of (a) $I_{\text {is }}$ in the experiment and (b) electron influx in the test region in the model as functions of $B_{\text {flat }}$.

for larger $B_{\text {flat }}$ both in the experiment and in the model. In the experiment, $t_{\text {fall }}^{\text {exp. }}$ exists during the flat section (orange hatch) but $t_{\text {fall }}^{\text {model }}$ does not exist during the flat section. This seems because the fall time is solely determined by the magnetic field profile in the model while it is also related to other conditions in the experiment. Figure 8 (a) also shows small difference in $B_{\text {prod. }}$ results in large differ-

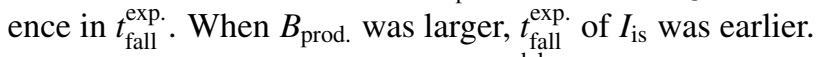
In contrast, as shown in Fig. 8 (b), $t_{\text {fall }}^{\text {mal }}$ does not depend on $B_{\text {prod. }}$. Except for the behavior in flat section, $t_{\text {fall }}^{\text {mod }}$ agrees

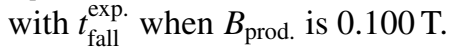

Figure 9 shows the magnetic field at the fall time of $I_{\text {is }}\left(B_{\text {fall }}^{\text {exp. }}\right)$ and electron influx in the test region $\left(B_{\text {fall }}^{\text {model }}\right)$ as functions of $B_{\text {flat }}$. As shown in Fig.9(a), $B_{\text {fall }}^{\text {exp }}$ seems to be proportional to $B_{\text {flat }}$ and is nearly equal to $B_{\text {flat }}$ when $B_{\text {prod. }}=0.103 \mathrm{~T}$. When $B_{\text {prod. }}=0.100 \mathrm{~T}, B_{\text {fall }}^{\text {exp. }}$ seems to be proportional to $B_{\text {flat }}$ for smaller $B_{\text {flat }}(<0.10 \mathrm{~T})$ but does not increase but rather decreases with $B_{\text {flat }}$ for larger $B_{\text {flat }}$ $(>0.10 \mathrm{~T})$. In contrast, as shown in Fig. 9(b), $B_{\text {fall }}^{\text {mol }}$ is nearly equal to $B_{\mathrm{ECR}}$ without depending on $B_{\text {flat }}$. Disagreement on $B_{\text {test }}$ at the end of the high $I_{\text {is }}$ phase between the model and the experiment was clearly shown in the new operation. 


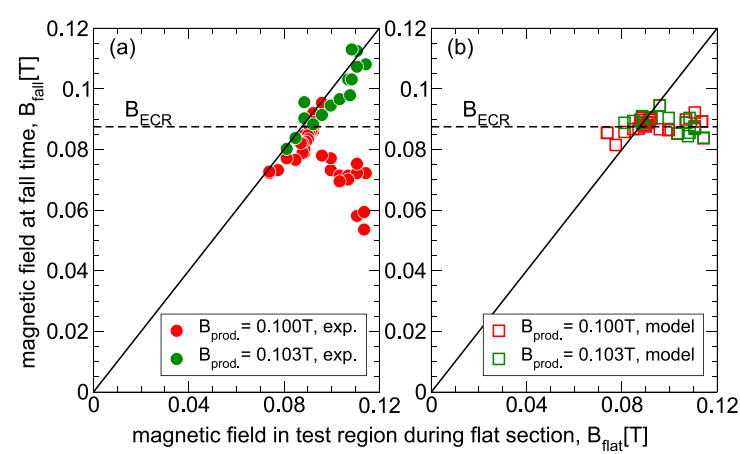

Fig. 9 Magnetic field at the fall time of (a) $I_{\text {is }}$ in the experiment and (b) electron influx in the test region in the model as functions of $B_{\text {latf }}$.

\section{Summary}

The time of beginning and end of the high density phase depended on the maximum magnetic field $\left(B_{\max }\right)$ applied in the downstream of a magnetic beach, so called the test region. When the $B_{\max }$ was higher, the duration of the high density phase was longer. In the model for the electron influx in the test region considering the magnetic mirror effect, the same tendency of the duration of the high density phase was obtained as in the experiment, but the magnetic field at decrease of the ion saturation current $\left(B_{\text {fall }}\right)$ was different.

To observe dependence on the magnetic field in the test region in detail, a new time evolution of magnetic field in the test region was developed to create a quasi-steady state or a flat section, $B_{\text {flat }}$. We investigated $B_{\text {flat }}$ dependence of the time $\left(t_{\text {fall }}\right)$ and magnetic field $\left(B_{\text {fall }}\right)$ at the end of the high density phase for different magnetic field in the production region $\left(B_{\text {prod. }}\right)$. Both in the experiment and in the model, $t_{\text {fall }}$ was later when $B_{\text {flat }}$ was larger. In the ex- periment, small difference in $B_{\text {prod. }}$ with only a few percent, $0.100 \mathrm{~T}$ and $0.103 \mathrm{~T}$, resulted in large differences in $t_{\text {fall }}$ and $B_{\text {fall }}$ for larger $B_{\text {flat }}(>0.10 \mathrm{~T})$. For smaller $B_{\text {flat }}(<0.10 \mathrm{~T})$, $B_{\text {fall }}$ seems to be proportional to $B_{\text {flat }}$ in both $B_{\text {prod. }}$ cases. For larger $B_{\text {flat }}(>0.10 \mathrm{~T}), B_{\text {fall }}$ still seems to be proportional to $B_{\text {flat }}$ in the $B_{\text {prod. }}=0.103 \mathrm{~T}$ case, but rather decreases with $B_{\text {flat }}$ in the $B_{\text {prod. }}=0.100$ T case. In the model, dependence of $t_{\text {fall }}$ on $B_{\text {flat }}$ agrees roughly to the experiment, but $B_{\text {fall }}$ was nearly equal to the value satisfying the electron cyclotron resonance condition.

Some discrepancies between the experiment and the model were newly found in the experiment with detailed scan in the magnetic field in the test region and in the production region with modified time evolution of the magnetic field in the test region. These findings suggest that there are some factors for formation of the high density phase that are not included in the present model. In the future, we will improve the model to reproduce the experiment and identify the formation mechanism of the high density phase. High density plasma supplied during this phase will enable us to study divertor plasma physics in an electron cyclotron plasma device.

\section{Acknowledgments}

The work was partly supported by MEXT/JSPS KAKENHI Grant Numbers JP19H01869, JP17H06231.

[1] N. Ohno et al., Nucl. Fusion 41, 1055 (2001).

[2] E.M. Hollmann et al., Phys. Plasmas 9, 1226 (2002).

[3] A. Okamoto et al., J. Nucl. Mater. 363-365, 395 (2007).

[4] H. Takahashi et al., Phys. Plasmas 23, 112510 (2016).

[5] Y. Zhang et al., Phys. Plasmas 15, 102112 (2008).

[6] M. Tanaka et al., J. Phys. Soc. Jpn. 60, 1600 (1991).

[7] D. Hamada et al., Plasma Fusion Res. 13, 3401044 (2018).

[8] A. Okamoto et al., Plasma Fusion Res. 14, 2401005 (2019). 